\title{
SAVOIRS ANTHROPOLOGIQUES, ADMINISTRATION DES POPULATIONS ET CONSTRUCTION DE L'ÉTAT
}

\author{
Benoît de L'Estoile, Federico NeIburg et Lygia Sigaud
}

L'ambition de ce numéro de la Revue de synthèse - à la croisée de la sociologie des savoirs scientifiques, de l'ethnographie de l'État et de l'histoire sociale de l'anthropologie - est d'analyser quelques aspects des relations entre la construction de l'anthropologie comme forme de connaissance de certaines populations et l'élaboration et la mise en œuvre par les États de politiques visant à administrer ces populations. Dans la division du travail entre disciplines scientifiques, l'anthropologie s'est spécialisée dans la description et la classification de groupes sociaux souvent désignés comme «primitifs », « arriérés », « marginaux », « tribaux », « sous-développés » ou « prémodernes », c'est-à-dire définis par leur extériorité et leur « altérité » par rapport au monde auquel appartiennent les anthropologues, lui-même défini par la «civilisation », le «progrès » scientifique et technique, la «modernité », l' «État». Cependant, l'identification (au sens fort de détermination de l'identité) de ces groupes sociaux «sans État» ou infra-étatiques (d'abord « races», « ethnies» ou « castes», puis «paysans », «pauvres » ou «minorités ») n'est paradoxalement devenue possible que parce que ceux-ci se trouvaient déjà soumis (ou en cours d'incorporation) aux États nationaux ou impériaux modernes, devenant l'objet de politiques spécifiques selon des orientations très diverses, pouvant aller de la préservation ou de la «protection » à des programmes de transformation sociale planifiée, en passant par des politiques répressives.

Les articles réunis ici traitent de lieux et de moments très variés : la place des populations indigènes dans la construction nationale au Mexique depuis le siècle passé, les débats classificatoires sur fond d'apartheid en Afrique du Sud, les projets de réforme de l'administration coloniale française, les politiques de l'État brésilien face aux populations indiennes et noires, le folklore dans la France de Vichy, la confrontation de l'architecture contemporaine à la réaffirmation culturelle kanak dans le Pacifique des années quatre-vingt-dix, la délimitation des terres indigènes dans le Revue de synthèse : $4^{\text {e }}$ S. n ${ }^{\text {os }}$ 3-4, juil.-déc. 2000, p. 233-263. 
Brésil d'aujourd'hui, ou les programmes contemporains de développement ${ }^{1}$. Dans ces divers contextes, des spécialistes des savoirs anthropologiques se trouvent en relation avec ceux qui sont chargés de mettre en place des politiques et avec les populations soumises à ces dernières. Dans ces situations, des groupes et des identités sociales sont définis, tels que les Indiens, les Noirs, les pauvres, les Kanak, les Ticuna, les Bushmen, les Mexicains ou les Français, mais aussi des entités comme l'État, l'Empire, la nation et la science.

Notre hypothèse est que ces trois éléments (savoirs anthropologiques, politiques d'État et populations) sont unis par une relation constitutive, au sens où chacun d'entre eux se définit par rapport aux autres. Dans les pages qui suivent, nous voudrions explorer, en nous appuyant sur les contributions ici réunies, les formes variées que prend cette relation dans diverses configurations et esquisser quelques pistes de réflexion qui surgissent de leur comparaison ${ }^{2}$.

DÉNONCIATION, ENGAGEMENT ET EXPERTISE

Les rapports entre l'anthropologie et l'État ont été évoqués depuis déjà longtemps dans certains textes qui abordent l'histoire de cette discipline. Une bonne partie de cette littérature relève d'un registre critique, dénonçant le fait que la discipline et ses praticiens auraient été «complices » de l'instauration et de la reproduction de formes coloniales ou néocoloniales de domination. Le rôle des anthropologues britanniques dans la conception et l'application de la politique d'Indirect Rule (administration indirecte) dans les colonies africaines dans l'entre-deux-guerres a fait l'objet de critiques particulièrement vives à partir de la fin des années soixante. De même, la participation d'anthropologues français à la gestion de populations colonisées (notamment en Algérie $^{3}$ ) et surtout le rôle, dans le contexte de la Guerre froide, d'anthropologues nord-américains dans la mise en place de politiques de contrôle et de domination en Asie du Sud-Est et en Amérique

1. Le projet de ce numéro a été conçu à l'issue du colloque franco-brésilien «Sciences sociales, État et société » (Rio de Janeiro, sept. 1997) qui a fait émerger l'intérêt d'une mise en relation des travaux présentés. Plusieurs d'entre eux, substantiellement remaniés, ont formé le noyau original de ce volume, complété par des contributions sollicitées par les organisateurs pour enrichir l'univers des comparaisons, sans pour autant prétendre à l'exhaustivité.

2. Tout en s'inspirant des travaux ici réunis et des discussions qui ont accompagné la préparation de ce numéro, ce texte n'engage que ses signataires.

3. Pour plus de précisions sur les références citées en notes, se reporter à la liste finale, p. 262-264. Ici, Lucas et Vatin, 1975; Le Mal de voir, 1976; Leclerc, 1972; Copans, 1975. 
latine ont également été dénoncés, parfois violemment ${ }^{4}$. À ces accusations de collusion entre l'anthropologie et les États impérialistes répondaient les protestations d'innocence des ethnologues accusés, mettant en avant l'absence d'intérêt, voire l'hostilité, que l'administration coloniale aurait manifestée à l'égard de l'ethnologie ${ }^{5}$.

La contrepartie d'une telle mise en cause était généralement la proposition - souvent explicitement présentée comme un programme d'action d'avant-garde - de faire de l'anthropologie l'instrument d'une politique alternative, s'opposant à la domination de l'État : une anthropologie engagée, au service des «intérêts des dominés ». Plus récemment, ces propositions ont refait surface sous la forme de plaidoyers en faveur d'une « anthropologie militante ${ }^{6} »$.

Sans vouloir minimiser l'importance pour la discipline de ces débats (auxquels ont pris part plusieurs des participants à ce numéro) et leurs enjeux éthiques et politiques, il est nécessaire de préciser que notre propos ne se situe pas à ce niveau. Notre intention n'est pas, en effet, de dégager ici des préceptes moraux ou des principes d'action (ce que doit ou ne doit pas faire l'anthropologue face à la « demande sociale »), mais de contribuer à construire, par le recours systématique à une approche comparative, des instruments d'analyse qui permettent de mettre ces discussions en perspective.

Certains articles, publiés dans ce volume, révèlent de façon exemplaire à la fois la complexité des relations entre pratiques savantes et politiques étatiques et le caractère récurrent des dénonciations dont elles font l'objet. Le texte d'Adam Kuper décrit ainsi l'émergence et la consolidation en Afrique du Sud d'une anthropologie bipolaire ${ }^{7}$. D'un côté, on trouvait des anthropologues qui s'efforçaient d'identifier et de classer des populations selon des critères raciaux et culturels, et proposaient de réguler les relations entre les unités sociales organisées par ces classifications en les maintenant séparées. Dans ce cas apparaît clairement la relation étroite entre une science classificatoire, qui met l'accent sur les différences entre groupes, et le projet de la ségrégation comme politique d'État — relation favorisée par la proximité sociale entre les anthropologues d'origine afrikaner, formés dans des universités de langue afrikaans, et les administrateurs de l'État d'apartheid. Inversement, cette proximité permet aussi de comprendre l'opposition des anthropologues de formation « anglophone » qui ont progressivement adopté une approche insistant sur l'unité fondamentale de la société sudafricaine, et qui accusaient leurs rivaux afrikaner de complicité dans la

4. WEAVER, 1973.

5. Goody, 1995; Loizos, 1977; voir aussi l'essai critique de Pels, 1997.

6. SCHEPER-Hughes, 1995.

7. Voir aussi KuPER, 1999b. 
mise en place de l'apartheid. Avec l'effondrement de celui-ci, dans les années quatre-vingt-dix, les héritiers de cette anthropologie critique ont été appelés à participer aux politiques d'unification et de refondation nationale de la nouvelle Afrique du Sud, redonnant une légitimité nouvelle à l'exercice de l'anthropologie au service de l'État.

Les brusques retournements de conjoncture politique constituent un moment favorable à l'exercice de la dénonciation. Dans le cas français, analysé dans l'essai de Florence Weber, la période de la Libération a ainsi vu la dénonciation d'un certain nombre de figures importantes de l'ethnologie de l'entre-deux-guerres ${ }^{8}$. Après 1944 , c'est même le «folklore », en tant que discipline, qui a été disqualifié pour avoir été associé au programme politique de l'État de Vichy, conduisant ses héritiers à redéfinir leur domaine comme «ethnologie de la France ». En même temps, la participation active de plusieurs ethnologues à la Résistance (notamment au sein du fameux «réseau du musée de l'Homme ») a contribué à valoriser une forme d'engagement dans la politique qui s'affirmera surtout pendant la guerre d'Algérie.

L'article de Claudio Lomnitz situe précisément l'apparition de la dénonciation comme arme dans la lutte entre les factions théoriques et politiques au sein de l'anthropologie mexicaine : ce fut en 1970 (peu après les grandes mobilisations étudiantes de 1968), avec la publication d'un livre qui voulait en même temps refonder la discipline et «démasquer » la complicité des anthropologues avec les politiques d'intégration des groupes indigènes dans la société mexicaine, en vigueur depuis les années quarante (l'indigénisme) ${ }^{9}$. Selon les auteurs, l'idéal de l'anthropologie après la révolution de 1910 (aider à la construction d'un Mexique métis, une nation indianisée) aurait abouti à sa mise au service d'une domination servant la reproduction d'un «capitalisme dépendant» et d'un «État autoritaire». Moins de vingt ans plus tard, plusieurs de ces rebelles, devenus des anthropologues reconnus, occupaient des positions centrales dans les institutions académiques et les organismes étatiques en charge de la gestion des populations indigènes. Comme le suggère Lomnitz, de la dénonciation de l'État à la cooptation par l'État de ceux qui le dénonçaient, la trajectoire de la génération de 1968 révèle un trait marquant de l'histoire de cette anthropologie nationale. Le recrutement d'anthropologues par les institutions de l'État afin d'élaborer, de réaliser et de légitimer scientifiquement les politiques de l'État se répète au cours du temps dans le processus de formation

8. Non seulement les folkloristes Georges-Henri Rivière et André Varagnac, mais aussi Henri Labouret, qui occupait jusque-là une position clé, enseignant l'ethnographie de l'Afrique à l'École coloniale et à l'Institut d'ethnologie.

9. Warman et al., 1970. 
d'un État national (le Mexique) dont un des piliers a été la construction de l'anthropologie comme savoir de l'État sur la société.

Cependant, l'incorporation de chercheurs dans la bureaucratie d'État n'est qu'un des aspects que prend la combinaison de la production de connaissances et de la mise en œuvre des politiques. Trois contributions explorent d'autres formes que peut aujourd'hui prendre cette relation sous la figure de l' expert $^{10}$. João Pacheco de Oliveira, engagé depuis longtemps dans la défense des droits fonciers des Indiens, évoque le rôle que les anthropologues se sont mis à jouer dans le processus de démarcation des terres indigènes au Brésil, après la nouvelle Constitution de 1988. À partir de ce moment s'est mis en place un nouveau processus légal, permettant la reconnaissance par l'État de groupes indigènes, reconnaissance qui fonde leur droit à la terre. L'identification et la territorialisation des groupes ethniques sont les deux versants d'un même processus où les anthropologues jouent un rôle central de médiateurs aux côtés des leaders indigènes, des avocats et des militants d'organisations non-gouvernementales (ONG), dont ils sont à la fois alliés et concurrents. Ici, l'anthropologue apporte son aide non seulement à l'État, mais aussi aux groupes indigènes qui ont besoin, pour exister juridiquement, de son savoir (reconnu par l'État en tant qu' «expertise» judiciaire). Pacheco de Oliveira invite ses collègues à prendre conscience de l'importance de cette pratique et de la redéfinition partielle du rôle de l'anthropologue qu'elle implique, plutôt que de la considérer comme un simple exercice d'anthropologie appliquée, marginal par rapport au travail proprement scientifique. Le témoignage d'Alban Bensa présente un cas original d'expertise anthropologique, au service à la fois d'un projet de l'État français et d'un mouvement de réaffirmation culturelle nationaliste qui cherche à s'émanciper de la tutelle de cet État désigné comme «colonial ». L'émergence, à partir de 1988, d'un processus de négociation entre le gouvernement français et le mouvement nationaliste kanak a abouti, entre autres, à la création d'une Agence pour le développement de la culture kanak, et du centre culturel Tjibaou. Renzo Piano, l'architecte de renommée internationale choisi pour le construire, chercha l'aide d'un anthropologue, espérant trouver, chez un spécialiste de la culture kanak qui s'était fermement engagé aux côtés des indépendantistes, le moyen de répondre au principal défi posé par le projet : objectiver le passé kanak (les relations des Kanak avec leur mémoire et les façons de se représenter leur tradition) et projeter la culture kanak vers le futur, respectant les aspirations émancipatoires. Comme Pacheco, Bensa évoque les questions

10. Le terme d' « expert » a pris parfois dans le contexte français une connotation péjorative, renvoyant implicitement à une accusation d'instrumentalisation de la science. Nous donnons, pour notre part, à « expertise » le sens purement descriptif de mobilisation d'une compétence socialement reconnue dans un domaine d'intervention de l'État. 
que ces situations inédites posent à l'ethnologue et à l'outillage théorique de sa discipline. Enfin, l'essai de Jorge F. Pantaleón aborde une situation professionnelle de plus en plus fréquente pour les anthropologues : celle de spécialistes des populations prises en charge par les programmes de développement, à la fois critiques vis-à-vis de ceux-ci, voire de la notion même de développement, et financés par les organisations interétatiques ou nongouvernementales chargées de mettre en œuvre ces programmes.

Au total, les appels à la responsabilité sociale des chercheurs et la dénonciation passionnée (ou le déni farouche) de la complicité des anthropologues avec le pouvoir d'État ont été — et continuent d'être — une des formes privilégiées qu'ont prises les affrontements au sein de la discipline et à ses frontières ${ }^{11}$. Ainsi, c'est souvent lors de polémiques qu'émergent les références à la relation entre savoirs anthropologiques, politiques publiques et populations. Dans ces divers cas, « engagement» et « récupération », « complicité » et « innocence », « conservatisme » et « progrès », «collaboration» et « résistance » ne sont pas des catégories analytiques mais bien plutôt des catégories indigènes du monde où agissent les anthropologues, utilisées par les agents intéressés à faire valoir leurs revendications et la légitimité de leurs actions. Si ces conflits favorisent une objectivation partielle des positions des adversaires, ils ne permettent pas la compréhension sociologique d'une relation qui ne doit pas être regardée seulement comme pathologique, mais comme structurelle ${ }^{12}$.

《SAVANT » ET « POLITIQUE 》

JUGEMENTS PRATIQUES ET CATÉGORIES D’ANALYSE

De façon plus générale, c'est souvent à l'occasion d'un retour réflexif des chercheurs sur leurs pratiques, sur les usages et les effets de leur savoir que sont évoqués les rapports entre la production de savoirs scientifiques et l'État. Dans un tel cas, la distinction entre «gens du savoir» (chercheurs) et «gens du pouvoir» («politiques » et «administrations ») est généralement prise aujourd'hui comme allant de soi, dans la mesure où elle correspond à l'existence d'univers professionnels différenciés qui possèdent des formations, des critères de hiérarchisation et de carrière distincts. Dans ces

11. En particulier, la redéfinition des frontières de l'anthropologie dans l'université nordaméricaine, confrontée au développement des «cultural studies », s'est pour une part importante effectuée autour d'une « critique » de l'anthropologie comme forme de domination (cf., p. ex., Marcus et Fischer, 1986).

12. Il serait naïf de prétendre être les premiers à prôner l'abandon d'une posture de dénonciation au profit d'un effort d'analyse. Voir, p. ex., Colonna, 1976. 
conditions, il est courant de poser le problème comme étant celui des relations entre «science» et «politique», ou encore, en reprenant le titre donné à la traduction française de deux conférences de Max Weber, Politik als Beruf et Wissenschaft als Beruf, entre le «savant » et le «politique ${ }^{13}$.

La formulation « le savant et le politique » est souvent utilisée pour penser le rapport entre les activités menées en tant que chercheur et en tant que citoyen, c'est-à-dire pour concilier les exigences, vécues comme potentiellement contradictoires, de l'appartenance à la communauté savante et celles de l'engagement dans les débats et les luttes politiques ${ }^{14}$. Cette approche - inspirée en fait, plus que par le propos de Weber, par la lecture qu'en fait Raymond Aron ${ }^{15}$ — fait l'économie d'une historicisation de cette question elle-même, en la posant comme un problème éthique et politique atemporel, qui doit être résolu sur le plan personnel. Dans cet usage, les figures du savant et du politique, loin d'être utilisées selon Weber de façon idéal-typique comme outils d'analyse, se trouvent bien plutôt mobilisées comme des archétypes dans une visée essentiellement prescriptive. Cette formulation, opposant deux figures constituées, le «savant (caractérisé par son appartenance à des institutions savantes professionnelles et l'adhésion à ses valeurs propres) et le « politique », vu comme « homme de pouvoir » (avec souvent une confusion entre les «professionnels de la politique » et les «professionnels de la bureaucratie »), paraît d'autant plus convaincante qu'elle correspond au sens commun des universitaires; elle peut donc être utilisée sur le mode de la connivence en laissant implicites un certain nombre de points. Ainsi, la référence rituelle à Weber risquet-elle paradoxalement de masquer l'absence de construction sociologique de la question, remplacée par des considérations politico-éthiques qui mêlent semi-objectivation de la position de ses adversaires, autojustification et discours prescriptif.

La discussion sur les objectifs et les moyens des sciences sociales et sur l'attitude à adopter face aux demandes des administrations publiques et des mouvements sociaux fait certes partie des débats que peuvent légitimement avoir les chercheurs. Dans ses écrits épistémologiques, Weber adopte explicitement une posture normative, en prônant l'exigence pour le chercheur de distinguer nettement entre la sphère des évaluations pratiques et

13. Weber, 1959. Dans le recueil de Gerth et Mills 1958, les deux textes sont également groupés sous le titre « Science and politics».

14. Pour un exemple récent de variation sur ce thème apparemment inépuisable, voir le dossier de Politix, 1999.

15. Raymond Aron, lui-même journaliste politique au Figaro et professeur à la Sorbonne depuis 1955, utilisait explicitement les conférences de Max Weber pour poser le problème des rapports entre «le savant et le politique » comme un dilemme pratique, voir Aron, 1959, p. 28 : «De là résulte, pour le professeur de sciences sociales qui veut être politiquement actif, une tension permanente. [...] Chacun de nous trouve sa réponse à ce problème personnel des rapports entre science et politique. » 
celle des analyses scientifiques ${ }^{16}$. Pourtant, Weber est très clair sur le fait que cette séparation représente à ses yeux un idéal, et non une description du monde savant. Quand une norme que nous considérons comme valide est prise pour objet d'analyse, elle doit être traitée selon les mêmes principes qu'une valeur que nous considérons comme fausse ${ }^{17}$. Il s'agit donc de l'analyser en tant qu'elle fait l'objet d'évaluations pratiques, c'est-àdire, en termes ethnographiques, comme une « catégorie indigène ».

Un tel principe de méthode invite donc à éliminer de l'analyse tout contenu normatif des termes «savant» et «politique », et à s'attacher exclusivement à leur usage en tant que catégories pratiques : abstractions produites et manipulées par les indigènes (ici, les chercheurs) dans leur effort pour construire le sens des relations entre le travail de chercheur et la mise en place de politiques étatiques.

L'essai critique de Florence Weber constitue un bon exemple des possibilités ouvertes par ce type d'analyse. Son point de départ est une interrogation sur la disparition simultanée du folklore scientifique et du régionalisme militant après 1945. Les travaux qu'elle analyse permettent d'avancer dans la mise en relation de faits rendus obscurs par la projection anachronique de catégories qui se sont établies après la guerre. Trois points sont centraux : d'abord, ni le folklore ni le régionalisme n'ont été inventés par Vichy - tous deux existaient auparavant sans qu'il y ait nécessairement coöncidence entre activité savante et adhésion à un programme politique; ensuite, juste avant la Seconde Guerre mondiale, le folklore était en cours d'institutionnalisation comme discipline scientifique; enfin, la période de Vichy, marquée par une rupture avec la façon républicaine de lier État, science et société, a paradoxalement pu favoriser dans certains cas une dissociation entre activités scientifiques et politiques. Ces éléments permettent à Florence Weber d'esquisser une interprétation historique qui, s'écartant radicalement de ceux qui veulent faire le tri entre «traitres » et « héros », décrit la façon dont la passion de la science a donné un sens aux actions de plusieurs individus engagés dans les études de folklore entre 1930 et 1945 - tout en adhérant à une définition de la science comme séparée de la politique.

Ce rappel historique invite à s'interroger sur les façons dont les relations entre l'univers de la science et celui de la politique sont aujourd'hui le plus souvent décrites et prescrites. Elles peuvent, pour simplifier, être réparties entre deux pôles. D'un côté, on trouve des discours, qui présentent la sphère du politique comme un moyen pour la science. Les chercheurs en sciences sociales seraient en mesure de distinguer, parmi leurs activités,

16. WeBER, 1992.

17. WeBER, 1992, p. 420-424. 
celles qu'ils effectuent en tant que «savants » (dans les universités et les institutions de recherche) et celles qu'ils réalisent en tant que «citoyens » ou « experts » (participant à la mise en œuvre par l'État de politiques), en utilisant stratégiquement les secondes au profit des premières. En ce sens, l'élaboration d'un projet de recherche financé par une administration, une ONG ou une fondation ${ }^{18}$ apparaît seulement comme une façon d'assurer les conditions de possibilité d'une connaissance scientifique. Le double résultat de cette activité (le rapport remis à la source de financement, et l'article publié dans une revue scientifique) est peut-être la meilleure illustration de la distinction entre «politique» et «science» qui caractérise cette conception. Au pôle opposé, on trouve la valorisation d'une science au service de la politique, les arguments sur la nécessité de rationaliser la solution des problèmes sociaux grâce à l'utilisation d'instruments de connaissance scientifiques, ou encore l'injonction à la recherche d'être engagée. Dans le premier cas, le public visé est celui des savants et la recherche pure est opposée à la recherche appliquée, qui se voit attribuer un statut mineur ${ }^{19}$. Dans le second cas, cette hiérarchie est inversée : le public principal est celui des hommes d'État, des militants ou des mouvements sociaux, et le résultat de la recherche tend à être polysémique, destiné à être lu autant par ceux-ci que par les chercheurs ${ }^{20}$.

Ces deux façons opposées de concevoir et de pratiquer les relations entre science et politique sont évidemment idéal-typiques. Dans les situations empiriques concrètes, rien n'empêche que les mêmes individus ou groupes utilisent en fonction du contexte l'un ou l'autre argument, ou adoptent une position intermédiaire, revendiquant le droit de travailler en scientifiques sur des problèmes pratiques.

«L'autonomie » de la science par rapport à la politique apparaît ainsi non pas comme une norme absolue (qui pourrait servir de critère invariant de classement des différents espaces scientifiques ou des divers individus au sein de ces espaces), mais comme une revendication produite, dans des conditions spécifiques, par certains agents et groupes. On peut donc s'interroger sur la pertinence d'un instrument analytique comme la notion d'autonomie. L'essai de Pantaleón pointe précisément son ambiguïté lorsqu'il est

18. Il faut souligner l'importance du rôle des grandes fondations nord-américaines (Rockefeller, Carnegie, Ford, Wenner-Gren) dans le développement du savoir anthropologique, que ce soit dans la Grande-Bretagne de l'entre-deux-guerres ou dans le Brésil depuis les années soixante-dix. Cet appui ne se limitait pas à l'anthropologie appliquée stricto sensu; il favorisait les recherches sur des problèmes considérés comme socialement importants.

19. Il est important de souligner que l'opposition entre «science pure » et « science appliquée » n'est pas une vérité immuable, mais une construction historique et instable.

20. Il faudrait ici analyser le rôle que jouent dans cette économie les publications situées à la frontière entre plusieurs mondes : revues savantes publiées par des ministères ou des « clubs de réflexion », revues « intellectuelles », publications à la fois militantes et universitaires liées à des mouvements de «minorités », etc. 
appliqué à l'étude de la constitution de l'univers des organisations nongouvernementales au Brésil, dans la mesure où l'autonomisation renvoie à la fois à un outil d'analyse des processus en cours et à un objectif affiché, ambiguïté d'autant plus marquée que plusieurs des analystes revendiquent une appartenance à la fois à l'univers des sciences sociales et au monde des ONG.

Au-delà de ce cas, un des apports de l'analyse comparative consiste précisément à montrer que cette valeur d'autonomie, loin de renvoyer à une réalité univoque et absolue, prend toujours un sens spécifique, défini par ce à l'égard de quoi l'autonomie est revendiquée : l'Église, le système universitaire, l'administration, les entreprises, etc. Comme le rappelle Steven Shapin, la définition de ce qui constitue la «science» et de ce qui est conçu comme lui étant « extérieur » (par exemple, la politique) ne peut pas être tenue pour acquise : elle est une construction qui varie au cours du temps ${ }^{21}$. Ainsi, une discipline peut conquérir son autonomie par rapport à d'autres savoirs en réalisant une alliance avec telle ou telle administration. D'un autre côté, les savants peuvent chercher à l'extérieur une plus grande autonomie vis-à-vis du contexte politique immédiat, développant ainsi ce qui peut apparaître comme une forme de «dépendance » à l'égard de la science étrangère.

Les valeurs de l'autonomie, de la «science pure » ou de la «science engagée » peuvent donc être soumises à l'analyse sociologique, tout en pouvant par ailleurs faire l'objet d'une adhésion. Pour contourner la difficulté évidente qu'il y a à prendre pour objet les valeurs attachées à notre propre position, et tenter de mettre à distance des prénotions que nous partageons en tant que membres de l'univers des savants, un des moyens est d'avoir recours à l'analogie. Le rapprochement entre producteurs intellectuels et clercs, souvent devenu un cliché, demeure cependant un outil de rupture précieux, en tant qu'il constitue le type idéal d'une forme spécifique de rapport entre un appareil bureaucratique et un type de production intellectuelle. On peut ainsi, en s'appuyant sur les analyses de Max Weber sur les rapports entre clercs et pouvoir temporel ${ }^{22}$, analyser la mobilisation du thème de la «science pure ». Au sein de l'espace religieux, c'est en effet au nom d'un idéal de "pureté » religieuse que des dénonciations de compromission avec le monde, le pouvoir et la richesse sont périodiquement faites, par des « outsiders» (par exemple, les réformes monastiques menées au nom d'un retour à une pureté perdue dans les compromis avec le monde séculier), mais aussi par le pouvoir hiérocratique central (le pape) voulant établir aux dépens des seigneurs laïcs son contrôle sur la distribu-

21. SHAPIN, 1992.

22. Voir Weber, 1996, notamment « Hiérocratie et pouvoir d'État». 
tion des charges ecclésiastiques. Dans cette perspective, une partie importante des valeurs constitutives de l'ethos universitaire apparaît structuralement homologue à celle de l'ethos clérical. Les clercs cherchent l'appui du pouvoir temporel, en y ayant notamment recours pour la défense de leur monopole contre les contestations diverses (les hérésies), tout en s'efforçant de garantir contre toute tentative d'immixtion leur autonomie, à la fois matérielle et intellectuelle, c'est-à-dire le contrôle sur l'accès aux charges et la définition de l'orthodoxie. Il est important de noter que la hiérocratie n'est pas pour autant nécessairement «extérieure » à l'État ${ }^{23}$. Dans cette relation, ce qui permet à la hiérocratie en tant que corps de défendre une certaine autonomie par rapport au pouvoir temporel, c'est qu'elle rend à celui-ci des services décisifs, en lui fournissant à la fois une légitimation et un certain nombre de «spécialistes » lettrés pour l'administration du royaume et la formation de ses élites.

Ainsi l'analyse que fait Max Weber des liens complexes entre pouvoir clérical et pouvoir temporel fournit-il un modèle qui permet de penser comme non contradictoires la revendication d'autonomie (et les conflits de frontière) et l'interdépendance, faite à la fois de solidarité et d'opposition structurelles, et d'analyser les différentes formes que prend historiquement cette relation. Un tel modèle invite, plutôt que de tenter de déterminer le degré plus ou moins grand d'autonomie des discours savants, à analyser les relations entre discours anthropologiques et pratiques de gestion des populations en fonction des transformations des formes d'interdépendance.

Là où le sens commun universitaire déplore les effets polluants de la «politique » sur la «science », l'analyse historique comparée permet de mettre en évidence une relation de dépendance mutuelle entre l'action de l'État et la production de connaissances sur les populations qu'il administre.

\section{L'ANTHROPOLOGIE, SAVOIR D'ÉTAT?}

La formulation en termes de « savant et politique » aboutit ainsi souvent à masquer l'existence de rapports constitutifs entre l'activité scientifique et l'État. En particulier, en focalisant l'interrogation sur les rapports entre l'activité d'un individu en tant que chercheur et son engagement politique

23. Le cas est particulièrement net (mais il n'est pas exceptionnel) dans les monarchies ibériques de l'époque moderne, avec le contrôle que le monarque exerce, par l'intermédiaire du «patronage royal» sur l'appareil ecclésiastique. Le Brésil impérial héritera de ce modèle, jusqu'à la séparation, en 1890, de l'Église et de l'État, sous le régime républicain. 
et sur les dilemmes moraux qui s'ensuivent, elle détourne l'attention d'un certain nombre de conditions de possibilité de la pratique scientifique, et, notamment, du rôle de l'État dans la création et le maintien des institutions dans lesquelles sont produites des connaissances.

Le point de vue que nous adoptons ici implique de considérer ensemble deux dimensions de la réalité sociale désignée par le terme État. D'un côté, l'État peut être analysé comme une entité qui «agit » dans le monde social en établissant des frontières ${ }^{24}$ : en identifiant des groupes, en reconnaissant des droits et en établissant des relations et des hiérarchies (entre les populations étudiées par les anthropologues), ainsi qu'en sanctionnant comme « authentiques » des formes de représentation collective (les « cultures » de ces populations). À cette dimension «durkheimienne» de l'analyse de l'État ${ }^{25}$, s'ajoute une autre dimension que l'on peut appeler « wébérienne». Dans ce cas, le terme État ne désigne pas un agent, mais un espace social où sont élaborées et mises en place des politiques à l'égard de certaines populations ou de certains «problèmes sociaux » et où interagissent des individus qui entretiennent des relations de concurrence et d'interdépendance. L'ensemble des textes réunis ici permet de montrer le caractère central que les luttes autour des frontières de l'État jouent dans les relations entre ces individus et dans la constitution des propriétés sociales et des valeurs morales qui légitiment leur existence sociale: savants «au service» de l'État ou «contre» l'État, au service des populations et des groupes sociaux dont l'existence dépend de la reconnaissance (juridique) par l'État; producteurs autonomes d'une connaissance «pure», qui se considèrent comme étant en relation d'opposition avec le «pouvoir d'État » - alors même qu'ils ont un statut de fonctionnaire, « assis sur des prébendes d'État», selon les termes de Max Weber ${ }^{26}$.

Plutôt que de concevoir a priori les «producteurs de connaissance» comme situés «à l'extérieur » de l'État, et donc comme confrontés à l'État comme un Autre, il est peut-être historiquement plus pertinent et sociologiquement plus fécond de les considérer comme partie prenante des luttes autour de la définition de ce qu'est l'État, de son rôle et des politiques qu'il doit mener. De ce point de vue, les conflits, par exemple entre tel anthropologue et tel fonctionnaire colonial, au lieu d'être interprétés en termes d'antagonisme entre «savants» et «politiques», peuvent être conçus

24. Bourdieu, 1994.

25. DuRKheIM, 1975.

26. On pourrait trouver une variante non étatique de cette situation dans les universités nord-américaines privées ou dépendant en partie de fonds privés, où des universitaires dont les chaires sont financées par les bénéfices réalisés à Wall Street peuvent se penser comme des rebelles en lutte contre le «système » ou l' « establishment ». 
comme une variante des conflits existant au sein de l'espace étatique, entre des agents de l'État appartenant à différentes administrations ${ }^{27}$.

L'article de Benoît de L'Estoile propose de distinguer de façon idéaltypique deux dimensions de la mobilisation de la science dans l'espace d'action de l'État : instrumentation et légitimation. D'un côté, les discours savants fournissent un certain nombre d'instruments de «mise en ordre » du réel qui peuvent informer des pratiques politiques, permettant notamment d'identifier les populations, de construire le sens de leurs actions, et de formuler les problèmes sociaux auxquels doivent répondre les politiques. D'un autre côté, la science fournit un ensemble d'arguments qui permettent de légitimer la proposition de certaines politiques. De façon plus fondamentale, l'appui donné par l'État aux institutions scientifiques lui permet de revendiquer une dimension rationnelle et objective, en manifestant qu'il a partie liée avec la science. Cette double dimension d'instrumentation et de légitimation est immédiatement visible dans le cas d'une discipline comme l'économie, dont les spécialistes ont construit leur identité professionnelle sur l'alliance de la sophistication théorique et de l'efficacité prédictive. Dans le cas de l'anthropologie, l'identité professionnelle aujourd'hui dominante valorise au contraire l'idéal de l'autonomie vis-àvis de l'État et de ses organes, ce qui amène à minimiser l'existence de relations, ou à ne les traiter que comme des déviations à éliminer.

Les analyses qui suivent permettent ainsi de remettre en question le paradigme du face-à-face entre deux univers nettement distincts, celui de l'anthropologie et celui de l'État, dont il s'agirait d'analyser les relations, en montrant qu'il est ici question d'une croyance qui rend mal compte de la diversité des situations empiriques. Il faut, en effet, rappeler que les savoirs sur les populations indigènes n'ont pas toujours été le monopole des anthropologues. Ceux-ci sont rarement les premiers à écrire sur les populations qu'ils étudient. Récits d'exploration, rapports de tournée, jugements de tribunaux indigènes, monographies de cercles, statistiques administratives, manuels d'instruction, travaux de commissions d'enquête, comptes rendus d'expéditions militaires, rapports politiques et ouvrages médicaux balisent un espace de savoirs, de discours et de pratiques au sein duquel le discours anthropologique se développe en entretenant avec eux une relation ambiguë.

L'article de Luiz Fernando Dias Duarte nous fait ainsi comprendre comment les recherches anthropologiques sur les Noirs au Brésil dans l'entredeux-guerres sont enracinées dans les débats sur la «question noire », et comment les propositions de politiques, notamment dans le domaine de

27. Voir, in Souza Lima, 1995, l'analyse exemplaire des conflits au sein de l'administration brésilienne pour comprendre les aléas des politiques indiennes. 
l'éducation, mobilisent des arguments savants. Une figure comme celle d'Arthur Ramos, médecin, psychiatre et anthropologue, est typique d'un espace caractérisé par une différenciation relativement faible, permettant au même homme d'être à la fois un savant reconnu dans des spécialités qui par la suite se sont autonomisées, et d'occuper une série de postes dans des institutions de psychiatrie et d'éducation, d'abord à Salvador, puis au sein de l'appareil d'État fédéral. Le développement des universités, à partir des années trente, crée les conditions d'émergence d'un nouveau corps de spécialistes, et Ramos s'éloigne de l'administration (tout en continuant à jouer un rôle dans la diplomatie culturelle) pour occuper la première chaire universitaire d'anthropologie du pays. Dans des configurations différentes, qu'il contribue à modifier, Ramos incarne donc successivement les figures du savant d'État, et de l'universitaire autonome ${ }^{28}$.

Loin de constituer uniquement une caractéristique archaïque du stade préinstitutionnel de la discipline, on peut penser que cette situation fournit une clé de lecture pour analyser la même relation dans d'autres configurations, où s'est réalisé un processus de différenciation disciplinaire. Partir de l'hypothèse que l'espace dans lequel se déploient les discours savants et les débats sur la politique des populations est, au moins en partie, commun, amène en particulier à ne pas présupposer comme acquise l'existence d'univers distincts; elle invite, au contraire, à analyser à la fois les passages entre discours savants et pratiques de pouvoir et le processus de différenciation, qui peut être analysé sous le signe de la division du travail et de la construction de monopoles.

Dans la France de l'entre-deux-guerres, la dynamique de concurrence entre puissances impériales et l'émergence de mouvements nationalistes suscitent le besoin d'une nouvelle légitimation de la domination coloniale. C'est alors que se développe une forme de domination des populations des territoires d'outre-mer où les sciences, et en particulier la nouvelle «science de l'homme» qu'est l'ethnologie, jouent un rôle important. S'appuyant sur la notion wébérienne de « domination rationnelle », l'article de Benoît de L'Estoile analyse diverses formes de convergence entre rationalité scientifique et rationalité bureaucratico-administrative. D'un côté, l'invention et la systématisation par l'administration coloniale de techniques d'identification et de compréhension des populations nourrissent l'élaboration d'un savoir «ethnologique» qui contribue à favoriser le

28. Si le parcours d'Arthur Ramos est exceptionnel, les passages, au cours d'une carrière, de l'université aux responsabilités administratives et politiques, et vice versa, ne sont pas rares encore aujourd'hui; le cas le plus notable au Brésil est celui de Fernando Henrique Cardoso, professeur à l'université de São Paulo dans les années 1960 et sociologue de renom international, aujourd'hui président de la République. On pourrait identifier dans d'autres contextes nationaux des carrières semblables, impliquant davantage d'autres disciplines comme le droit ou l'économie. 
développement d'une grille de lecture « ethnique » de la réalité indigène. De l'autre, les arguments pour une «action coloniale fondée en raison » sont développés en parallèle à l'établissement d'une alliance entre ceux qui, autour de l'École coloniale, entendent se définir comme spécialistes de l'administration des indigènes, et ceux qui veulent s'établir comme spécialistes de la science des indigènes, autour de l'Institut d'ethnologie et du musée de l'Homme. La rationalisation de la domination aboutit ici à un processus simultané de création de spécialités et de spécialistes qui construisent dans la relation de dépendance et de concurrence qui les unit leur identité et leur spécificité.

L'article d'Antonio Carlos de Souza Lima analyse le rôle qu'a eu, dans la restructuration des organismes étatiques chargés d'élaborer et de mettre en œuvre au Brésil la gestion des populations indigènes, l'importation du modèle mexicain de l'indigénisme, qui présupposait une participation active des anthropologues à la politique des populations. La création de la Section d'études du Service de protection des Indiens (SPI), puis du musée de l'Indien, fournit un cas paradigmatique de création au sein d'un appareil administratif d'un organe spécifiquement préposé à la production et à la diffusion du savoir sur les populations indigènes. De ce point de vue, on peut la rapprocher de la création de services anthropologiques au sein de l'administration de certaines colonies britanniques ou françaises, d'une section au sein du Department of Native Affairs en Afrique du Sud, ou d'un Office des recherches scientifiques coloniales. Ces différents cas sont autant d'exemples d'un processus de spécialisation fondé sur la division du travail et l'alliance entre des spécialistes du savoir et des techniciens de la gestion des populations ${ }^{29}$.

Cet ensemble de cas permet à son tour d'analyser comme des variantes les configurations où l'administration (ou certains de ses secteurs) apporte son appui au développement d'institutions autonomes spécialisées dans la production de savoir sur les populations indigènes : musées et expéditions ethnographiques, départements universitaires, instituts de recherche, sociétés savantes.

En effet, l'existence d'une discipline consacrée à l'exploration des différences entre populations établit une confirmation scientifique de la nécessité d'une politique qui s'adapte à ces différences. Réciproquement, la nécessité d'élaborer des politiques spécifiques en fonction de leurs caractéristiques constitue un argument de poids en faveur du développement d'une connaissance scientifique de ces populations ${ }^{30}$.

29. Cette situation est aussi celle de l'Institut national de la statistique et des études économiques (INSEE), de l'Institut national d'études démographiques (INED), de l'Institut brésilien de géographie et de statistiques (IBGE) ou de la Direccion general de estadisticas au Mexique.

30. Inversement, l'opposition aux théories différentialistes au fondement de l'Indirect Rule ou de la politique de développement séparé en Afrique du Sud implique souvent une dénon- 
On observe donc un processus de consolidation mutuelle entre l'affirmation de groupes qui construisent leur identité sociale et professionnelle en tant que spécialistes de l'administration de certaines populations spécifiques (indigénistes, administrateurs des colonies, officiers des affaires indigènes, Service de protection des Indiens, native administrations, etc.) et l'émergence d'un discours savant prenant pour objet ces populations. Autrement dit, l'existence de l'anthropologie en tant que «science des indigènes » valide le projet d'élaborer des «politiques indigènes » qui justifient l'existence d'agents spécialisés et contribuent en retour à singulariser les « populations indigènes » soumises à ces politiques — ce qui en fait un objet privilégié pour les études ethnologiques. Ce processus circulaire aboutit donc au renforcement de la différence ${ }^{31}$. Cet intérêt commun constitue le fondement d'une alliance (qui n'exclut pas les conflits de frontière) entre deux types de "spécialistes des indigènes », ceux qui les administrent et ceux qui les étudient. Cette alliance se manifeste notamment dans l'appui donné par certains secteurs de l'État aux efforts de construction par les anthropologues d'un monopole du savoir sur les populations indigènes. Le travail de reconstruction historique effectué par Souza Lima fait ainsi apparaître un constat qui n'est paradoxal qu'en apparence : l'autonomisation de l'anthropologie comme discipline universitaire au Brésil, surtout à partir des années soixante, se comprend seulement si on prend en compte la participation des anthropologues à la formulation des politiques de l'État.

Depuis les fonctionnaires écrivant des travaux ethnographiques sur les populations qu'ils administrent jusqu'aux universitaires bénéficiant de contrats de recherche avec des ministères ou des ONG ou participant à la formation de spécialistes de la gestion des populations, en passant par les membres d'instituts de recherche rattachés à l'administration, on a donc une vaste gamme de positions qui impliquent des formes différentes d'interdépendance, sans être réductibles à une même échelle de comparaison, et encore moins à un critère idéalisé d'autonomie. Cette variété de configurations favorise une circulation plus ou moins intense entre savoirs

ciation de l'anthropologie. Voir L'Estorle, $1997 \mathrm{~b}$ et l'article d'Adam KuPER, dans ce numéro, p. 278-279.

31. Affirmer l'existence d'une telle relation de constitution mutuelle entre les savoirs anthropologiques, les politiques de l'État et les populations constituées en tant qu'objets à la fois de ces politiques et de ces savoirs n'est pas sombrer dans un constructivisme absolu. Il n'est pas question, par exemple, d'affirmer que les populations qui vivaient sur ce qui deviendra le territoire du Brésil n'étaient pas différentes des Européens qui les conquérirent, mais il s'agit de reconnaître que c'est le fait de la Conquête, et la mise en place par l'État colonial d'une législation indienne, qui unifie des groupes extrêmement hétérogènes sous la catégorie d'Indiens, et constitue le substrat sur lequel a pu se développer beaucoup plus tard une discipline «ethnologique » spécifiquement consacrée aux populations indigènes. 
anthropologiques et savoirs administratifs, qui contribue à produire des formes d'objectivation du monde social.

Un des points qui émerge dans plusieurs contributions à ce numéro est le lien entre l'anthropologie et l'élaboration et la stabilisation de grilles de lecture de la diversité des populations. Kuper et Lomnitz montrent comment, en Afrique du Sud ou au Mexique, les catégories désignant les groupes de population étaient à l'origine variables en fonction des contextes locaux. Les exigences combinées de la mise en place d'un appareil statistique unifié et d'une standardisation des classifications scientifiques, à une époque où l'anthropologie était conçue comme une «science naturelle des sociétés », ont souvent contribué à cristalliser les identités des populations dominées.

Kuper montre ainsi le jeu complexe d'élaboration et de transformation des catégories de population en Afrique du Sud et au Botswana : catégories d'autodésignation et appellations données par les groupes voisins, parfois reprises comme dénominations administratives et savantes, passage de catégories fondées sur le statut à des catégories fondées sur la race, hésitations entre fondement biologique ou linguistique pour la détermination des limites des groupes, interactions entre classifications statistiques et catégories juridiques, abandon de dénominations jugées dépréciatives, etc. Recensements de populations, cartes et fichiers ethniques, musées et films ethnographiques, encyclopédies tribales contribuent à ce processus de cristallisation et de transformation, non pas au sens où ils créeraient de toutes pièces la réalité, mais dans la mesure où ils contribuent à donner ou non la sanction de la science et du droit à certains groupes, qui peuvent devenir le fondement de nouvelles identités collectives. Les textes de Pacheco de Oliveira et de Bensa invitent à s'interroger sur le rôle que jouent aujourd'hui encore les anthropologues dans la cristallisation et l'officialisation d'identités ethniques ou culturelles, alors même que les transformations des rapports de force entre les groupes dont sont originaires les anthropologues et les groupes qu'ils étudient ont des effets sur le type de savoir produit, et sur les transformations des catégories sociales.

Cette « affinité élective », fondée sur la division du travail entre production de connaissances et administration, est cependant compliquée par le fait que les anthropologues se sont souvent attribué le rôle de porte-parole des populations qu'ils étudient. Bronislaw Malinowski défendait ainsi l'idée que l'anthropologue devait devenir «non seulement l'interprète de l'indigène, mais aussi son champion ${ }^{32} \gg$. Une telle situation a été favorisée par le fait que ces populations étaient le plus souvent «sans voix », et que sur le terrain l'anthropologue est régulièrement considéré par ceux auprès

32. Malinowski, 1937. 
de qui il enquête comme un «médiateur » qui pourra intervenir « en haut lieu » pour répercuter une demande ou favoriser l'accès à telle ou telle ressource (crédits, médicaments, eau, protection, honneurs, etc.). En ce sens, les cas de Manuel Gamio au Mexique, engagé dans un programme de «transformation sociale » à Teotihuacan, ou de Marcel Griaule, obtenant de l'administration coloniale française la création d'un barrage pour les Dogon, doivent être considérés comme poussant à la limite une logique d'assistance aux populations.

Aujourd'hui, les transformations des groupes étudiés, et notamment leur scolarisation croissante, modifient en profondeur les interactions des anthropologues avec ceux chez qui ils effectuent leur « terrain ${ }^{33}$ ». De plus en plus, l'anthropologue doit négocier sa présence et faire face aux interrogations légitimes de ceux qui lui demandent quel profit ils tireront de sa recherche. Il serait réducteur de n'attribuer qu'à cette seule exigence l'attitude des anthropologues qui se font les relais des revendications de ceux qu'ils étudient, mais c'en est sans doute une des dimensions.

Cette mission de défense des « populations » joue un rôle important dans la construction de l'anthropologie en tant que groupe professionnel. Les associations professionnelles, telles l'American Anthropological Association (AAA), l'Association of Social Anthropologists britannique (ASA), l'Associação Brasileira de Antropologia (ABA), etc., se donnent explicitement le rôle de promouvoir l'anthropologie auprès des pouvoirs publics, et se sont constituées en groupes de pression intervenant dans la définition des politiques de l'État pour garantir les droits des populations dominées qu'ils étudient ${ }^{34}$. Dans le cas du Brésil, les anthropologues ont notamment joué, à la fin des années quatre-vingt, un rôle très important pour obtenir la reconnaissance des droits des Indiens dans la nouvelle Constitution alors en discussion $^{35}$, mais aussi pour tenter de faire avancer le processus de Réforme agraire.

Les anthropologues ne sont cependant pas les seuls candidats à ce rôle de médiateurs entre les populations et les organes étatiques ou internationaux. Ils se trouvent en concurrence avec les représentants d'autres disciplines (sociologues, psychologues, économistes, politistes ou juristes) ou d'autres groupes professionnels (médecins, avocats, religieux), des militants politiques ou syndicaux, des ONG, des organisations indigènes, etc.

33. Voir, à propos du Brésil, Albert, 1997.

34. Ainsi, l'ABA possède actuellement trois commissions explicitement chargées de suivre l'évolution de certaines questions politiques: une commission des Affaires indigènes, une commission des Droits de l'homme et une commission de la Terre.

35. Cf., p. ex., Carneiro da Cunha, 1987, p. 11-12: «Ce livre veut être avant toute chose un instrument pour les membres de l'Assemblée constituante [...] quand ils s'occuperont de la question indigène. [...] Pour assurer les droits d'une population sans défense est nécessaire une législation forte, sans ambiguïtés, [...] qui ne puisse pas être dévoyée. » 
Les anthropologues apparaissent ainsi comme un des groupes qui sont en concurrence pour définir le rôle de l'État et les politiques que celui-ci a à mener. Ce qui est en jeu, c'est non seulement la capacité des anthropologues à faire reconnaître leur monopole de la connaissance scientifique de certaines populations, mais aussi leur capacité à définir les problèmes sur lesquels l'action de l'État doit s'exercer ${ }^{36}$.

Si les savoirs anthropologiques ont partie liée avec la construction de l'État, on peut penser qu'il y a un lien entre le type d'État qui est construit et les formes variées que prennent les savoirs anthropologiques dans différents contextes nationaux.

ANTHROPOLOGIES NATIONALES ET ESPACE INTERNATIONAL

L'ensemble des cas abordés ici permet en effet d'analyser la situation de l'anthropologie dans les différents contextes nationaux en fonction de trois principes de variation :

$1^{\circ}$ la nature des unités politiques (État-nation ou État-empire) dans lesquelles elle se développe, et à la construction desquelles elle contribue;

$2^{\circ}$ l'articulation différenciée entre l'émergence et le maintien de traditions nationales spécifiques et la circulation internationale des théories scientifiques et des modèles de gestion des populations;

$3^{\circ}$ la position occupée dans l'espace international et les transformations au cours du temps du système d'interdépendance entre États.

L'expression « anthropologies nationales » peut prendre deux acceptions sensiblement différentes, selon que l'adjectif « national» renvoie à une distinction entre État-nation et empire, ou à une opposition entre ce qui relève de la sphère nationale et de l'espace international ${ }^{37}$. Dans le premier sens, l'historien de l'anthropologie George W. Stocking opposait ainsi, au sein de la tradition euro-américaine, les anthropologies nationales tournées vers la nation (anthropologies of «nation-building ») aux anthropologies développées dans les métropoles en lien avec la constitution des empires (anthropologies of «empire-building $)^{38}$. Ainsi, le caractère des études anthropologiques en Grande-Bretagne — qui fournit le paradigme des anthropologies impériales — aurait été principalement déterminé par la

36. Ainsi, les anthropologues, proches de Bronislaw Malinowski et de l'International Institute of African Languages and Cultures, ont joué un rôle essentiel dans la reformulation de la politique coloniale britannique en Afrique dans les années trente, notamment en contribuant à donner un nouveau contenu à la notion de développement. Voir L'Estoile, 1997c.

37. Voir, notamment, Gerholm et Hannerz, 1983.

38. Stocking, 1983, p. 172. 
rencontre des « Autres » à la peau foncée dans l'empire d'outre-mer, alors que dans nombre de pays d'Europe continentale, c'est la relation de l'identité nationale et de l'altérité intérieure qui aurait joué le rôle essentiel ${ }^{39}$. Les anthropologies nationales se borneraient à étudier les populations présentes sur le territoire national, tandis que les anthropologies des métropoles auraient une extension bien supérieure.

Dans un second usage, la notion «d'anthropologie nationale » caractérise une anthropologie définie par son caractère local par opposition à une «anthropologie internationale », composée d'anthropologues d'origines diverses, mais dont le «centre » est surtout constitué par l'anthropologie anglo-américaine et, dans une moindre mesure, française. Du point de vue du centre qu'assume explicitement Stocking, les anthropologies nationales apparaissent comme des formes résiduelles destinées à disparaître au sein d'une anthropologie internationale.

Ces analyses ont, dans un premier temps, le mérite de rappeler que la belle formule selon laquelle la science ne connaît pas de frontières recouvre bien des inégalités (notamment du point de vue linguistique) et que la circulation des idées ne supprime pas les rapports de force. Cependant, le passage constant et souvent implicite du premier au second sens a pour effet pervers de renforcer une dichotomie qui rend équivalents national et périphérique d'un côté, international, impérial, «metropolitan» et central de l'autre ${ }^{40}$.

La distinction entre anthropologies nationales et impériales a l'intérêt d'attirer l'attention sur le caractère plus ou moins central, dans divers types de communautés politiques, des populations qui sont objets des savoirs anthropologiques : le «problème indigène » ne se pose évidemment pas avec la même acuité s'il concerne les populations de lointaines possessions ou si, comme en Afrique du Sud ou au Mexique, ces populations sont au cœur de l'espace défini comme «national », même si elles sont maintenues dans une position dominée. Ainsi est-on amené à faire l'hypothèse que l'anthropologie est appelée à jouer un rôle d'autant plus grand que la diversité des populations pose problème à ceux qui définissent les politiques de l'État. De fait, il semble, d'après les cas étudiés ici, que l'on puisse établir une homologie approximative entre le domaine d'étude des anthropologues et le champ d'action de l'unité politique à laquelle ils appartiennent. Autrement dit, les populations sont constituées comme objets de savoir dans la mesure où elles sont constituées comme enjeux des politiques de l'État.

39. Voir, en ce sens, les nombreux exemples donnés par THIEsSE, 1999.

40. Il est caractéristique que dans le numéro d'Ethnos, organisé par Tomas Gerholm et Ulf Hannerz, consacré aux « anthropologies nationales », l'univers des cas étudiés se limite à la « périphérie » : Brésil, Inde, Pologne, Suède, Soudan, Canada, Québec, le tout étant couronné par un «point de vue du centre » demandé à un membre du département d'anthropologie de Chicago, l'un des plus réputés internationalement, voir Stocking, 1983. 
C'est au sens où elle est étroitement liée à la construction de l'Étatnation que l'on peut, avec Lomnitz, caractériser l'anthropologie mexicaine comme une « anthropologie nationale » — ou, pour utiliser un néologisme forgé par Norbert Elias, «natiocentrique ${ }^{41}$ »- caractérisée par deux aspects : d'un côté, le rôle central de l'anthropologie dans l'élaboration de l'idéologie du nationalisme mexicain et la mise en œuvre de politiques de l'État fondées sur cette idéologie; de l'autre, la création de cadres institutionnels par le gouvernement depuis la fin des années 1930. L'École nationale d'anthropologie et histoire (ENAH) a longtemps conservé le monopole de la formation des anthropologues, fournissant l'essentiel des cadres de l'establishment anthropologique mexicain; l'Institut national d'anthropologie et histoire (INAH) est à la fois une des principales institutions de recherche anthropologique, et un des lieux d'élaboration et de diffusion de l'image de la nation valorisée par le nationalisme mexicain (synthèse entre métissage et modernité) et répandue dans tout le pays par les musées d'anthropologie et d'histoire, en particulier le Museo Nacional de Antropología de Mexico. Enfin, l'Institut national indigéniste (INI) est l'organe de l'État le plus important consacré à l'élaboration et à la mise en œuvre de la politique de gestion des populations indigènes, et offre aux anthropologues un de leurs principaux débouchés.

Conformément à la définition du champ d'application de l'anthropologie aux populations habitant le territoire national, les « anthropologues nationaux » identifient les frontières de l'État (ou de l'Empire) avec les limites de leurs objets de recherche. Ainsi, Kuper montre comment les anthropologues sud-africains ont borné leurs analyses des groupes ethniques aux populations qui étaient à l'intérieur des frontières de l'État, sans prendre en compte celles qui se trouvaient plus au nord, alors que celles-ci se reconnaissaient parfois elles-mêmes comme leur étant apparentées. On peut ainsi voir à quel point s'applique aux anthropologues l'affirmation de Max Weber, selon laquelle les intellectuels ont contribué de façon décisive à la construction de ces formations politiques que nous appelons « nations » et à la formation des «sentiments nationaux » et de la «conscience nationale ${ }^{42}$.

Le sentiment national est parfois étroitement associé au sentiment impérial. Deux cas sont particulièrement intéressants pour analyser les interactions entre « anthropologie nationale » et « anthropologie impériale ». Le

41. Elias, 1989, parle de «natiozentrische Denkorientierung ». Cette caractéristique n'est pas propre aux seules idéologies nationalistes, mais à toutes les théories de la culture ou de la société qui ont comme horizon une ambition descriptive et prescriptive, en lien avec les idées de «belle culture » et de «société bonne», tout en se référant toujours implicitement à ces unités sociales et culturelles appelées nations.

42. WeBER, 1968, vol. II, p. 925-926. 
Portugal présente ainsi le cas d'une anthropologie à la fois impériale, nationale et périphérique ${ }^{43}$. Le nationalisme portugais se construit, en effet, autour de l'idée que la grandeur du Portugal réside essentiellement dans le fait que c'est un peuple de conquérants et de colons. Le paradoxe du Portugal est qu'il a été à la fois le premier pays européen à se constituer un empire outre-mer, et qu'il occupe une position marginale parmi les puissances européennes. L'ethnologie portugaise du $\mathrm{xx}^{\mathrm{e}}$ siècle est donc marquée par la préoccupation de mettre en valeur à la fois la diversité des populations et l'unité de cette nation-empire, et par le souci de démontrer que le Portugal se trouve au «niveau international» du point de vue scientifique.

« Nation» et «empire » apparaissent ainsi non comme deux catégories exclusives, mais comme deux pôles dont se rapproche plus ou moins à tel moment telle anthropologie. Plus un État a une capacité de projection à l'extérieur (sous forme coloniale ou «hégémonique»), plus ses anthropologues sont susceptibles d'aller sur le terrain hors des frontières nationales. Une telle hypothèse permet d'échapper à l'essentialisation qui guette les réflexions en termes de centre et de périphérie, en reliant les transformations que connaissent certaines anthropologies à l'évolution du système d'interdépendance entre États.

Le second cas est celui des États-Unis où une anthropologie nationale se transforme en une anthropologie impériale et internationale. Au cours des années 1930, les anthropologues affirment la capacité de leur discipline à résoudre des problèmes pratiques, portant surtout leur attention sur la diversité sociale et culturelle des États-Unis : Indiens, Noirs et immigrants de diverses origines, petites communautés rurales et grandes villes rassemblant une population fortement différenciée. Les «problèmes pratiques » auxquels entendait répondre "l'anthropologie appliquée » étaient liés au processus d'homogénéisation sociale et culturelle qui marque la constitution des États nationaux : santé, éducation, alimentation et travail définissaient les principaux domaines où ce processus était en cours. Le centre d'intérêt se trouvait à l'intérieur des frontières nationales, l'application de l'anthropologie constituant essentiellement un thème de politique interne ${ }^{44}$.

La participation des anthropologues à l'effort de guerre américain pendant la Seconde Guerre mondiale constitue une étape cruciale dans la projection internationale de l'anthropologie nationale américaine. Comme l'ont montré Marcio Goldman et Federico Neiburg, l'invention d'un nouvel objet théorique pour la discipline, les études du caractère national, sanctionne l'existence d'un nouveau domaine d'application de l'anthropologie :

43. Ribeiro Thomaz, 2000.

44. La première réunion de la Society for Applied Anthropology eut lieu à Harvard, en mai 1941, signalant l'émergence d'un nouveau domaine. 
la politique extérieure ${ }^{45}$. Dès 1939, quelques-uns des anthropologues de l'école «Culture et personnalité » (Margaret Mead, Gregory Bateson et Geoffrey Gorer) fondent le Committee for National Morale avec l'objectif de produire une réflexion sur la culture américaine qui permette de faire des recommandations pour conserver élevé le moral américain pendant le conflit. Après Pearl Harbor, les membres du Committee travaillèrent pour l'Office of War Information, lié à la Marine nord-américaine, rejoints notamment par Ruth Benedict, Clyde Kluckhohn, Ralph Linton, George P. Murdock et Rhoda Métraux. Si, jusque-là, le Committee s'intéressait essentiellement aux États-Unis et à la culture américaine, il chercha aussi les moyens de parvenir à une meilleure compréhension des nations alliées, mais surtout ennemies. Le résultat de ces enquêtes n'était pas seulement destiné aux dossiers des organes de renseignements des forces armées, ni à conseiller la formulation de la politique extérieure américaine, mais fut également jugé selon des critères académiques, présenté à des congrès, publié en articles dans les revues de la discipline, transformés en livres qui voulaient établir un nouvel objet pour l'anthropologie : le « caractère natio$\mathrm{nal}^{46} \gg$.

Mead définissait comme suit les possibles « applications pratiques » de l'étude des «cultures à distance », à laquelle le contexte de guerre avait obligé les anthropologues :

«The approach described in this Manual has been used for a variety of political purposes: to implement particular governmental programs within a country, to facilitate relationships with allies, to guide relationships with partisan groups in countries under enemy control, to assist in estimating enemy strengths and weaknesses, and to provide a rationale for the preparation of documents at the international level. All these uses involve diagnosing the cultural regularities [...] The diagnosis is made for the purpose of facilitating some specific plan or policy, and at least implicitly, includes predictions of expected behavior that may make such a plan or policy successful or unsuccessful ${ }^{47}$. 》

Ainsi, le domaine d'études des anthropologues américains s'élargit-il à un rythme similaire à celui de la puissance américaine. Dès les années trente, les anthropologues étaient présents dans la zone d'influence américaine: au Mexique, dans les Caraïbes et le Pacifique. Après la

45. Neiburg et Goldman, 1998. Cet article a été présenté lors du colloque de Rio.

46. Le cas le plus célèbre est le livre de Ruth Benedict sur le caractère japonais, publié en 1946, voir BENEDICT, 1989.

47. Mead et Métraux, 1953, p. 397. Il s'agit d'un manuel que Margaret Mead et Rhoda Métraux présentèrent en 1949 au Columbia University Research in Contemporary Cultures (inauguré deux ans avant par Ruth Benedict grâce à un financement de la Human Research Division. Office of Naval Research). 
Seconde Guerre mondiale, l'anthropologie américaine se fait à l'échelle mondiale ${ }^{48}$.

Cette relation entre le domaine d'intervention d'un État et le domaine d'étude de ses anthropologues explique les fortes asymétries constatées : de fait, comme le souligne Lomnitz, les anthropologues américains étudient le Mexique, mais la réciproque n'est pas vraie. La sphère d'activité des anthropologues mexicains se limite aux domaines constitués en enjeux pour l'État mexicain : ainsi, les anthropologues mexicains qui ont fait des recherches aux États-Unis ont essentiellement étudié les émigrants mexicains.

Comme le souligne cet exemple, les contextes nationaux dans lesquels se structure la relation entre l'anthropologie et l'État ne sont jamais des isolats. Au contraire, la construction des États nationaux (et impériaux) doit être analysée comme un processus à la fois interne et externe, dans une situation d'interdépendance concurrentielle entre États, engagés dans une compétition pour le statut et le prestige. Plusieurs contributions révèlent le caractère déterminant des préoccupations de légitimation vis-à-vis des autres puissances. La politique de chaque État à l'égard de ses populations «périphériques » ou de ses «minorités » fait, en effet, l'objet d'une évaluation dans l'espace international. En tant que « science des indigènes », l'anthropologie est souvent mise à contribution pour justifier l'intérêt des États pour leurs populations indigènes ou «minorités ethniques ». Lomnitz et Souza Lima rappellent ainsi que l'exportation du modèle indigéniste d'association entre anthropologie et État a fait partie de la politique étrangère du Mexique, à côté de la mise en scène de l'archéologie précolombienne. De même, on peut mettre en relation le développement des savoirs anthropologiques comme «science des indigènes » en France et en Grande-Bretagne dans l'entre-deux-guerres avec la nécessité de démontrer internationalement l'intérêt des puissances coloniales pour les indigènes ${ }^{49}$. Dans le cas de la France, l'ethnologie est définie par ses promoteurs comme une science coloniale, tournée vers l'identification et la compréhension des populations indigènes; en même temps, elle se trouve investie d'une mission nationale, au sens où elle manifeste aux yeux du monde entier l'universalisme scientifique du pays et son respect pour les sociétés indigènes qu'il domine. Le souci du prestige national et la crainte d'être « dépassé » par les autres puissances dans l'étude des populations indi-

48. La relation n'est pas mécanique : elle est médiatisée par les financements de projets de recherche sur des zones «stratégiques ». À propos des effets de cette ouverture de nouveaux espaces (comme l'Indonésie) sur la carrière d'un anthropologue comme Clifford Geertz, voir KUPER, 1999a, p. 77-81.

49. L'Estoile, 1997a et 1997c; voir, aussi, du même, l'article, p. 291-323, dans ce numéro. 
gènes de l'empire jouent ainsi un rôle considérable dans l'appui donné par l'État aux institutions anthropologiques ${ }^{50}$.

Une telle approche historique des interdépendances amène à analyser les processus de circulation internationale d'individus, de théories et de technologies politiques, et la constitution «d'écoles nationales ${ }^{51}$ » en considérant à la fois les intérêts qui favorisent l'internationalisation et les intérêts à l'affirmation de la dimension nationale. L'internationalisation peut en effet représenter une ressource, notamment à travers les alliances qu'elle permet, mais aussi une menace, dans la mesure où elle peut remettre en cause le monopole interprétatif des intellectuels nationaux.

Comme le suggère Souza Lima, l'association de certains anthropologues brésiliens avec l'indigénisme mexicain a contribué à renforcer leur position dans l'espace anthropologique national, et leur présence dans l'élaboration et la mise en place de politiques dirigées vers les populations indigènes. L'indigénisme fait l'objet non seulement d'une «importation », mais aussi d'une réappropriation, voire d'une nationalisation, en fonction des traditions de gestion des populations indigènes développées au Brésil, pour une part héritées de la colonisation portugaise. Un des enjeux de la création d'une anthropologie nationale (dont un des lieux d'émergence est le Musée national) est précisément de ne pas laisser aux savants étrangers (notamment allemands) le monopole de l'étude des groupes indigènes. Très récemment encore, Pacheco de Oliveira montre que la pression internationale (en particulier, celle des ONG) joue un rôle fondamental dans la mise en place du processus de démarcation des terres indiennes, auquel les anthropologues sont appelés à participer ${ }^{52}$.

Dias Duarte met nettement en évidence une dynamique similaire. Comme au Mexique, les intellectuels brésiliens de la première moitité du $\mathrm{xx}^{\mathrm{e}}$ siècle sont préoccupés à la fois par la construction de la nation brésilienne (dont un des enjeux essentiels est la place de la population noire) et par sa valorisation à l'extérieur ${ }^{53}$. Dias Duarte montre comment on passe progressivement, dans l'entre-deux-guerres, d'une problématique de la «civilisation » du Brésil - horizon qui permet de comprendre les formes spécifiques que prennent l'importation et la réinterprétation des théories

50. Aujourd'hui encore, on peut penser qu'un des enjeux de la construction d'un « centre culturel kanak », confiée à un des grands noms de l'architecture contemporaine, est aussi lié à des considérations de prestige de la France dans le Pacifique, accusée par les voisins de la Nouvelle-Calédonie d'être une des dernières puissances coloniales.

51. Crawford, Shinn et Sörlin, 1993.

52. Une organisation non-gouvernementale consacrée à la défense des minorités ethniques comme Survival International, qui compte de nombreux anthropologues parmi ses membres, joue ainsi un rôle tout à fait important dans la mobilisation des opinions publiques internationales.

53. Sur les rapports entre anthropologie et construction de la nation au Brésil, voir PEIRANO, 1981 et 1992. 
psychanalytiques et de l'œuvre de Lucien Lévy-Bruhl - à une incorporation de l'héritage afro-brésilien dans la construction d'une culture nationale brésilienne. Dans ce contexte, la psychanalyse fait l'objet d'une lecture non seulement du point de vue de la psychologie individuelle, mais aussi d'un point de vue politique, dans la mesure où elle est perçue comme permettant de penser le triomphe de l'intellect sur l'instinct, conçu comme une métaphore des élites éclairées guidant les masses dominées par des pulsions irrationnelles.

En Afrique du Sud, alors que l'anthropologie afrikaner, confrontée à un environnement international largement hostile, devient de plus en plus nationaliste dans une alliance étroite avec l'État d'apartheid, les anthropologues anglophones, dominés politiquement, jouent au contraire la carte de l'internationalisation, à tel point que certains, ayant quitté leur pays natal, ont obtenu un renom international en tant que membres de l'École britannique $^{54}$ et non en tant que sud-africains.

Ainsi, l'affinité entre le «natio-centrisme » plus ou moins explicite des «anthropologies nationales », et le fait que celles-ci soient utilisées dans l'élaboration et la mise en œuvre de politiques internes n'implique en rien qu'il s'agisse d'anthropologies isolées, ou de créations authentiquement nationales comme le voudraient les idéologues nationalistes. Au contraire, le sens de l'expression « anthropologie nationale » ne se comprend pleinement qu'en le reliant à l'univers des relations internationales, à la fois en tant qu'espace de circulation des idées et des modèles et en tant que système d'interdépendance concurrentielle entre unités politiques.

\section{SAVOIRS LOCALISÉS ET PERSPECTIVE COMPARATIVE}

Nous voudrions, pour terminer cette présentation, revenir sur l'intérêt du va-et-vient entre démarche historique et analyse du présent, puis entre études de cas et comparaison. Il n'est pas indifférent que l'ensemble des auteurs de ce numéro revendiquent leur appartenance disciplinaire à l'anthropologie (même si certains d'entre eux se définissent aussi, selon les contextes, comme sociologues ou historiens). La plupart des contributions, même celles qui portent sur une période historiquement éloignée, se donnent pour objectif d'analyser des processus qui permettent de mieux comprendre le présent.

Chacune des contributions de ce volume est donc localisée et datée, au double sens où elles portent sur des unités circonscrites spatialement et

54. Isaac Schapera, Meyer Fortes, Max Gluckman ont quitté l'Afrique du Sud et poursuivi l'essentiel de leur carrière en Grande-Bretagne. 
temporellement, et où leurs auteurs sont situés socialement et historiquement, par leur trajectoire professionnelle et intellectuelle, par les positions qu'ils occupent dans leur espace national comme dans leurs institutions de rattachement, par les préoccupations et les formes de rhétorique qui dominent dans les univers de discussion auxquels ils appartiennent, etc. Chacun des participants à ce numéro se trouve engagé dans des débats et des conflits politiques, académiques et théoriques, locaux, nationaux et internationaux. La sociologie des intellectuels la plus élémentaire autorise à penser que les trajectoires et les positions institutionnelles et politiques ne sont bien évidemment pas sans lien avec la façon dont sont construits les textes de ce numéro, celui-ci inclus. Tous possèdent une dimension, plus ou moins explicite, d'auto-analyse, dans la mesure où il s'agit d'exercices de réflexivité sur des trajectoires individuelles ou collectives avec lesquelles les auteurs entretiennent des relations de proximité plus ou moins grandes.

Pourtant, cela n'implique pas — même si seul le lecteur pourra en juger - que le présent volume ne soit qu'une suite d'interprétations plus ou moins «subjectives». La perspective comparative a été présente tout au long de l'élaboration de ce numéro, et les auteurs se sont efforcés de respecter la règle d'écrire pour un lecteur non familier des contextes locaux. Cette contrainte, d'avoir pour interlocuteur imaginaire des lecteurs « étrangers » aux enjeux politiques et institutionnels immédiats, constitue en un sens un équivalent pratique de l'injonction de Max Weber, qui demandait aux chercheurs de produire un raisonnement qui puisse être accepté comme valide par un Chinois, c'est-à-dire précisément par quelqu'un censé pouvoir évaluer sa cohérence sans partager aucun des implicites et des conventions admises ${ }^{55}$. Même si une telle modalité d'écriture reste de l'ordre de l'idéal, la confrontation entre des cas éloignés dans l'espace et dans le temps permet de mettre à distance les enjeux immédiats et de relativiser certains jugements qui, dans chacun des univers pris séparément, associent sur le mode de l'évidence telle formulation théorique ou tel point de vue sur les politiques des populations à des positions sur l'échiquier politique (« de droite » ou « de gauche »). Par exemple, le fait de prendre pour base d'une politique le postulat de la diversité des populations n'est pas intrinsèquement «conservateur» ou «progressiste», mais prend son sens en fonction des configurations historiques variables. Ainsi, la reconnaissance du fait qu'un groupe possède une «culture » originale et des traditions qui le singularisent vis-à-vis d'autres populations avec lesquelles il est en interaction peut-elle être associée aussi bien à une position politique «de gauche », comme dans le cas des Kanak de Nouvelle-Calédonie, ou à une position « réactionnaire », comme dans l'Afrique du Sud de l'apartheid.

55. WeBER, 1992, p. 130-131. 
La comparaison permet ainsi de neutraliser partiellement un certain nombre de jugements d'ordre normatif, en faisant apparaître des traits structurels : replacés au sein d'un univers de configurations, l'engagement des anthropologues pendant la Seconde Guerre mondiale dans le combat contre les puissances de l'Axe ou aujourd'hui dans la lutte pour le développement (généralement évalué positivement) et leur implication dans la gestion des populations colonisées ou l'élaboration des classifications de l'apartheid (évaluée négativement) deviennent susceptibles d'être étudiés au sein du même cadre analytique, qui permet d'en faire ressortir à la fois les similarités et les différences. Cela n'invalide en rien la possibilité de porter un jugement d'ordre politico-éthique sur tel ou tel épisode de l'histoire de la discipline, mais permet d'en faire une lecture sociologique.

Nous espérons que les contributions ici rassemblées pourront fournir aux anthropologues (et aux autres) des éléments de réflexion sur les liens entre pratiques savantes et politiques des populations en évitant de prendre pour paradigme implicite l'état actuel des rapports entre chercheurs, commanditaires de recherche sur des thèmes d'actualité et débats publics, mais en éclairant la spécificité des situations des chercheurs de tel ou tel domaine.

Ce que permet la double démarche d'historicisation et de comparaison, c'est donc un décentrement du point de vue : au lieu d'appréhender la relation entre la production de savoirs et la construction des politiques uniquement à partir de sa propre position, en supposant une séparation constitutive entre la Science et l'État, il s'agit de substituer le modèle d'un espace commun au sein duquel se constitueraient de façon progressive à la fois un ensemble de savoirs et un ensemble de politiques de gestion des populations qui, tout en se différenciant au cours du temps, restent en relation de façon multiple. On observe donc un processus de construction mutuelle entre l'État en tant qu'appareil de gestion des populations perçues comme différentes et en tant qu'instrument d'unité nationale, les savoirs anthropologiques et les populations elles-mêmes. Bien entendu, une telle situation, loin d'être propre à l'anthropologie, semble caractériser l'ensemble des sciences sociales : c'est souvent en lien avec les mouvements de réforme sociale visant une transformation ou une extension de l'intervention de l'État dans le domaine social que se sont constituées les sciences sociales ${ }^{56}$.

Cette prise en compte de l'inscription des sciences sociales dans le champ d'action des différents États fournit également une piste permettant de comprendre l'émergence de «traditions nationales » distinctes. S'il est en effet nécessaire, pour comprendre les particularités nationales de

56. Voir, p. ex., les analyses réunies in WAgner, Wittrock et Whitley, 1991, et in RueschMEYER et SKOCPOL, 1996, ainsi que plusieurs ouvrages recensés dans ce numéro. 
l'anthropologie, d'analyser la place variable qu'elle occupe dans les divers systèmes de disciplines, d'enseignement et de recherche (liens étroits avec la philosophie ou la préhistoire ici, avec l'archéologie ou la linguistique là) ${ }^{57}$, les multiples formes d'interdépendance avec l'État au sens où on l'a défini ici, jouent un rôle essentiel dans la constitution d'anthropologies nationales.

L'histoire des sciences sociales, ainsi entendue, constitue une contribution autant à l'anthropologie des pratiques classificatoires et des processus d'identification qu'à la sociologie historique de l'État. Ces considérations préliminaires n'ont d'autre ambition que d'inviter le lecteur à mettre en œuvre lui-même, en découvrant les études qui suivent, la démarche comparative esquissée ici.

\author{
Benoît DE L'EstoILE \\ Laboratoire de Sciences sociales \\ École normale supérieure \\ 48, bd Jourdan, \\ F-75014 Paris \\ estoileb@elias.ens.fr
}

\author{
Federico NeIBURG \\ PPGAS/Museu Nacional \\ CEP 20940-040 \\ Rio de Janeiro, Brésil \\ fnmv@alternex.com.br
}

\author{
Lygia Sigaud \\ PPGAS/Museu Nacional \\ CEP 20940-040 \\ Rio de Janeiro, Brésil \\ lsigaud@alternex.com.br
}

57. Whitley, 1984. 


\section{LISTE DES RÉFÉRENCES}

Albert (Bruce), 1997, «Situation ethnographique et mouvements ethniques. Réflexions sur le terrain post-malinowskien », in Michel AGIER, dir., Anthropologues en dangers. L'engagement sur le terrain, Paris, Jean-Michel Place, p. $75-88$.

Aron (Raymond), 1959, «Introduction », in Max Weber, Le Savant et le politique, Paris, Plon.

Benedict (Ruth), 1989, The Chrysanthemum and the sword. Patterns of Japanese culture, $1^{\text {re }}$ éd. 1946, ici New York/Boston, Houghton Mifflin Co.

Bourdieu (Pierre), 1994, «Esprits d'État. Genèse et structure du champ bureaucratique », in ID., Raisons pratiques. Sur la théorie de l'action, Paris, Seuil, p. 101-133.

Carneiro da Cunha (Manuela), dir., 1987, Os Direitos do Índio. Ensaios e documentos, São Paulo, Brasiliense Comissão Pró-Índio de São Paulo.

Colonna (Fanny), 1976, «Production scientifique et position dans le champ intellectuel et politique. Deux cas : Augustin Berque et Joseph Desparmet », in Le Mal de voir, p. 397-414.

Copans (Jean), 1975, Anthropologie et impérialisme, Paris, Maspero.

Crawford (Elizabeth), Shinn (Terry) et Sörlin (Sverker), éd., 1993, Denationalizing science. The contexts of international scientific practice, Dordrecht, Kluwer (Sociology of the Sciences Yearbook).

Durkheim (Émile), 1975, «L'État», in ID., Textes. T. III : Fonctions sociales et institutions, Paris, Minuit, p. 172-178.

Elias (Norbert), 1989, «Introducción » (Prologue à l'édition allemande de 1968), in El Proceso de la civilización. Investigaciones sociogenéticas y psicogenéticas, Mexico, Fondo de Cultura Economica, p. 9-46.

Elias (N.), 1996, The Germans. Power struggles and the development of habitus in the nineteenth and twentieth centuries, ed. by Michael SCHRÖTER, transl. from the German by Eric Dunning et Stephen Mennell, New York, Columbia University Press.

Gerholm (Tomas) et Hannerz (Ulf), 1983, « The shaping of national anthropologies », Ethnos, vol. XLVII, 1, p. 5-35.

Gerth (Hans H.) et Mills (C. Wright), éd., 1958, From Max Weber. Essays in sociology, $1^{\text {re }}$ éd. 1946, ici New York, Oxford University Press.

Goody (Jack), 1995, The Expansive Moment. Anthropology in Britain and Africa, 1918-1970, Cambridge, Cambridge University Press.

KUPER (Adam), 1999a, Culture. The anthropologist's account, Cambridge, Harvard University Press.

KUPER (A.), 1999b, « South African anthropology. An inside job », in ID., Among the anthropologists. History and context in anthropology, Londres, Athlone Press, p. 145-170. 
LECLERC (Gérard), 1972, Anthropologie et colonialisme, Paris, Fayard.

Le Mal de voir. Ethnologie et orientalisme: politique et épistémologie, critique et autocritique..., 1976, Paris, Union générale d'éditions (Cahiers Jussieu, 2, Université de Paris VII, 10-18).

L'Estolle (Benoît de), 1997a, "Africanisme et "Africanism". Esquisse de comparaison franco-britannique », in L'Africanisme en questions, sous la dir. d'Anne Piriou et d'Emmanuelle Sibeud, Paris, Centre d'études africaines, École des hautes études en sciences sociales (Dossiers africains), p. 19-42.

L'Estorle (B. de), 1997b, « Au nom des "vrais Africains". Les élites scolarisées de l'Afrique coloniale face à l'anthropologie (1930-1950) », Terrain, numéro spécial « Miroirs du colonialisme», 28, p. 87-102.

L'Estolle (B. de), 1997c, « The "natural preserve of anthropologists". Anthropology, scientific planning and development», Information sur les sciences sociales, vol. XXXVI, 2, juin, p. 343-376.

LoIzos (Peter), dir., 1977, «Anthropological research in British colonies. Some personal accounts », Anthropological Forum, vol. IV, 2.

LuCas (Philippe) et Vatin (Jean-Claude), 1975, L'Algérie des anthropologues, Paris, Maspero.

Malinowski (Bronislaw), 1937, «Preface », in Julius Lips, The Savage hits back, Londres, Lovat Dickson.

Marcus (George H.) et Fischer (Michael M. J.), 1986, Anthropology as cultural critique. An experimental moment in the human sciences, Chicago, University of Chicago Press.

MeAd (Margaret) et MéTRaux (Rhoda), éd., 1953, The Study of culture at a distance, Chicago, Chicago University Press.

Neiburg (Federico) et Goldman (Marcio), 1998, « Anthropology and politics in studies of national character », Cultural Anthropology, vol. XIII, 1 .

Peirano (Mariza G.), 1981, The Anthropology of anthropology. The Brazilian case, thèse de doctorat, Cambridge, Harvard University.

Peirano (M. G.), 1992, Uma antropologia no plural. Três experiências contemporâneas, Brasília, Editora da UnB.

Pels (Peter), 1997, «The anthropology of colonialism. Culture, history and the emergence of Western governmentality », Annual Review of Anthropology, vol. XXVI, p. 163-183.

Politix, 1999, «Les savants et le politique », 48, p. 1-206.

Ribeiro Thomaz (Omar), 2000, Ecos do Atlântico Sul. Representações sobre o terceiro império português, Rio de Janeiro, Editora da UFRJ/FAPESP.

Rueschemeyer (Dietrich) et Skocpol (Theda), éd., 1996, States, social knowledge, and the origins of modern social policies, Princeton/New York, Princeton University Press/Russell Sage Foundation.

SchePER-Hughes (Nancy), 1995, «The primacy of ethical. Propositions for a militant anthropology », Current Anthropology, vol. XXXVI, 3, p. 409-420.

Shapin (Steven), 1992, "Discipline and bounding. The history and sociology of science as seen through the externalism-internalism debate », History of Science, 30.

Souza Lima (Antonio Carlos de), 1995, Um grande cerco de paz. Poder tutelar, indianidade e formação do Estado no Brasil, Petrópolis, Vozes.

Stocking (George W.), 1983, "Afterword. A view from the center», Ethnos, vol. XLVII, 1, p. 172-186.

Thiesse (Anne-Marie), 1999, La Création des identités nationales. Europe, XVIII ${ }^{e}$ $X x^{e}$ siècle, Paris, Seuil. 
Wagner (Peter), Wittrock (Björn) et Whitley (Richard), éd., 1991, Discourses on society. The shaping of the social science disciplines, Dordrecht, Kluwer (Sociology of the Sciences Yearbook).

Warman (Arturo), Bonfil (Guillermo), Nolasco (Margarita), Olivera (Mercedes) et Valencia (Enrique), 1970, De eso que llaman antropología mexicana, Mexico, Editorial Nuestro Tiempo.

WeAver (Thomas), éd., 1973, To see ourselves. Anthropology and modern social issues, Glenmview, IL/Londres, Scott, Foresman and Co.

Weber (Max), 1959, Le Savant et le politique, introd. par Raymond Aron, Paris, Plon.

WEBER (M.), 1968, Economy and society. An outline of interpretative sociology, éd. par Gunther Rотн et Claus Wiтtich, Berkeley, University of California Press, 2 vol.

WeBer (M.), 1992, Essais sur la théorie de la science (tirés de Gesammelte Aufsätze zur Wissenschaftslehre, Tübingen, Mohr, 1951), Paris, Plon/Presses Pocket.

Weber (M.), 1996, Sociologie des religions, éd. et trad. de l'allemand Jean-Pierre Grossein, introd. Jean-Claude Passeron, Paris, Gallimard (Bibliothèque des sciences humaines).

Whitley (Richard), 1984, The Intellectual and Social Organization of the sciences, Oxford, Clarendon Press. 